\title{
INTEGRAL results on the January 2009 activity from the Anomalous X-ray Pulsar 1E 1547.0-5408
}

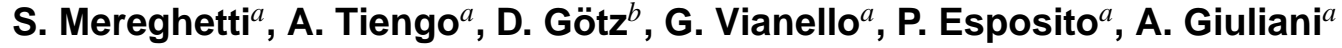 \\ aINAF-IASF Milano, \\ via E. Bassini, 15, I 20133 Milano, Italy \\ E-mail: sandro@iasf-milano.inaf.it \\ ${ }^{b}$ CEA-Saclay, DSM/IRFU/SAp, \\ Orme des Merisiers, Bat.709,F 91191 Gif-sur-Yvette, France
}

In January 2009, many short bursts of soft gamma-rays were detected from the direction of the Anomalous X-ray Pulsar 1E 1547.0-5408 by different satellites. Here we report on the observations obtained with the INTEGRAL SPI-ACS detector during the period with the strongest bursting activity. More than 200 bursts were detected at energies above $80 \mathrm{keV}$ in a few hours on January 22. Among these, two remarkably bright events showed pulsating tails lasting several seconds and modulated at the $2.1 \mathrm{~s}$ spin period of 1E 1547.0-5408. The energy released in the brighter of these two bursts $\left(\sim 10^{43} \mathrm{erg}\right.$ for $\left.\mathrm{d}=5 \mathrm{kpc}\right)$ was smaller than that of the three giant flares seen from soft gamma-ray repeaters, but higher than that of typical bursts from soft gamma-ray repeaters and anomalous X-ray pulsars. The large fluence emitted in the soft X-ray range during this bursting episode, gave rise to bright dust scattering rings observed in the following days.

The Extreme sky: Sampling the Universe above $10 \mathrm{keV}$ - extremesky2009,

October 13-17, 2009

Otranto (Lecce) Italy 


\section{Introduction}

Soft gamma-ray repeaters (SGRs) are X-ray sources characterized by the emission, during sporadic periods of activity, of short ( $<1 \mathrm{~s})$ bursts of soft gamma-rays with peak luminosity up to $10^{42} \mathrm{erg} \mathrm{s}^{-1}$, and, much more rarely, by the occurrence of giant flares, releasing up to $10^{46} \mathrm{ergs}$, or possibly more. SGRs are well explained as magnetars: isolated neutron stars powered by extremely high magnetic fields, $B>10^{14}-10^{15} \mathrm{G}$ [1]. Short bursts have been detected also from most Anomalous X-ray Pulsars (AXPs), thus supporting their interpretation as another class of magnetars. The most recent observations of AXPs and SGRs suggest that their different classification merely reflects the way they were originally discovered. A recent review of AXPs and SGRs is given in [2].

1E 1547.0-5408 was discovered almost 30 years ago [3], but only recently it was proposed as a member of the AXP class [4]. It is a transient X-ray source located in the supernova remnant candidate G327.24-0.13. Its spin period $(P=2.1 \mathrm{~s})$ was discovered in the radio band and later seen also in X-rays [5]. The period derivative, $\dot{P}=2.3 \times 10^{-11} \mathrm{~s} \mathrm{~s}^{-1}$, gives, with the usual dipole assumption, a magnetic field of $2.2 \times 10^{14} \mathrm{G}$. The emission of several SGR-like bursts in October 2008, as well as the more spectacular bursting activity displayed by the source in January 2009, completed the picture of 1E 1547.0-5408 as a typical magnetar.

Here we discuss the observations of 1E 1547.0-5408 obtained with the INTEGRAL SPI-ACS instrument during the January 2009 bursting period, as well as follow-up observations carried out with Swift and XMM-Newton. A more detailed account of these results is published in $[6,7]$.

\section{Observation of bursts with INTEGRAL}

The Anti-Coincidence Shield (ACS) of the INTEGRAL SPI instrument, besides serving to veto the background in the germanium spectrometer, is routinely used as a nearly omni-directional detector for gamma-ray bursts [8]. It produces light curves with a resolution of $50 \mathrm{~ms}$ for photon energies $\gtrsim 80 \mathrm{keV}$, but without energy and directional information.

Thanks to the highly elliptical orbit of INTEGRAL, the ACS was the only detector providing a continuous, uninterrupted coverage of 1E 1547.0-5408 from 14:35 UT of January 20 to 04:23 UT of January 23, 2009. The ACS light curve covering the most intense bursting period is displayed in Fig. 1. The strong activity shown on January 22, with the emission of hundreds of bursts in a time span of a few hours $[6,9,10]$, resembles similar episodes observed from other SGRs and AXPs (see, e.g., $[11,12,13])$. The peak of the bursting rate occurred around 6:48 UT, when more than 50 bursts were recorded in 10 minutes. The high fluence emitted in this short time interval is responsible for the expanding dust scattering halos discovered in Swift/XRT X-ray images obtained about one day later [7].

Two bursts had durations longer than the spin period of 1E 1547.0-5408 and clearly showed a modulation at $2.1 \mathrm{~s}$ (see Fig. 2). They occurred at 6:48:04 UT and 8:17:29 UT. The first burst was brighter, it started with a very bright $(\sim 0.3 \mathrm{~s})$ and short initial spike, followed by a $\sim 8 \mathrm{~s}$ long pulsating tail. These features of the light curve are typical of giant flares from SGRs. Therefore it is interesting to compare the energetics of this burst with that of the three historical giant flares and with other strong bursts and flares from magnetars. 


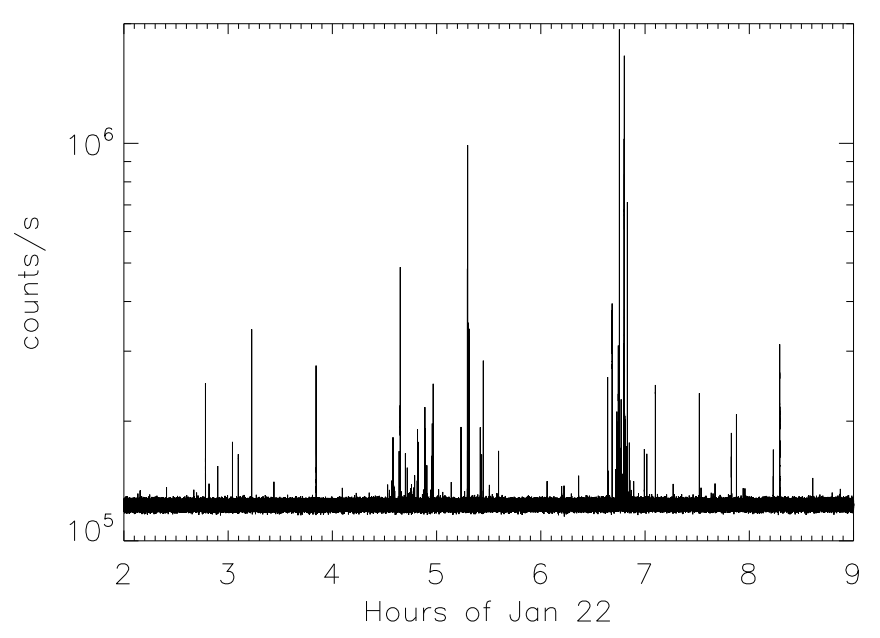

Figure 1: Light curve of 1E 1547.0-5408 at E $>80 \mathrm{keV}$ obtained with the SPI-ACS during the most active period on 2009 January 22 . The time binning is $50 \mathrm{~ms}$.
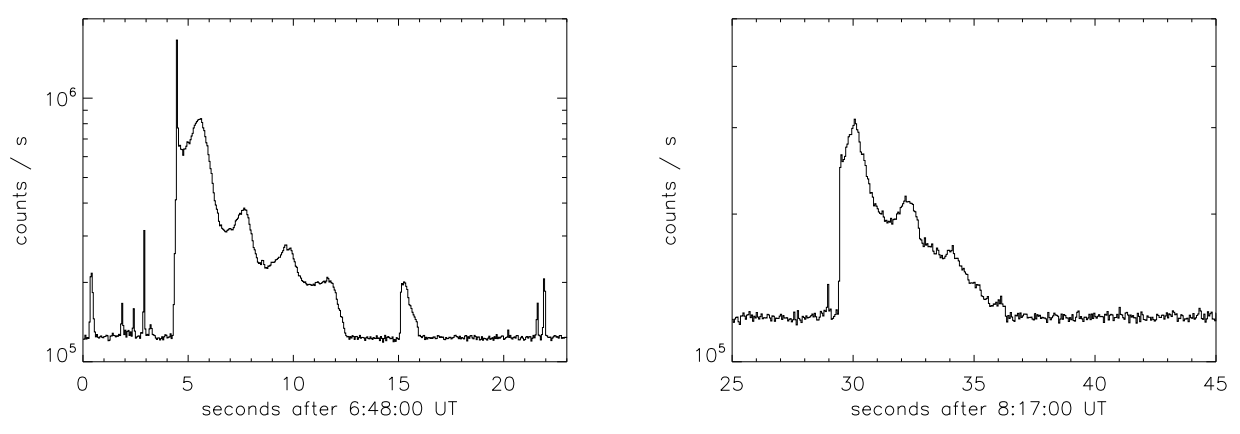

Figure 2: SPI-ACS light curves of the two longest bursts seen from 1E 1547.0-5408 on 2009 January 22. Note that the initial spike of the 6:48:04 UT burst was brighter than shown in the figure, due to the non negligible instrumental dead-time at high count rates.

This is done in Fig. 3, where the plot axis refer to the energy in the short initial spikes and in the pulsating tails. Note that in some cases only lower limits to the emitted energy could be derived due to instrument saturation. The giant flares are the points in the upper right part of the plot. Other noteworthy events from AXPs and SGRs are also plotted, including a few short bursts without tails and the few cases of pulsating tails where a bright initial spike was not detected. It is clear from Fig. 3 that there is a large spread of intensities, from the typical short bursts to the brightest giant flares.

The energy released in the pulsating tails of the giant flares of SGR 0526-66, SGR 1900+14, and SGR 1806-20 was much larger than the value of $5 \times 10^{42}(\mathrm{~d} / 5 \mathrm{kpc})^{2}$ erg we derived for 1E 1547.0-5408. However, the difference is mainly due to the shorter duration of the 1E 1547.05408 pulsed tail. In fact the tails following the three historical giant flares lasted a few minutes, but their average luminosity $\left(\sim 3 \times 10^{41}-10^{43} \mathrm{erg} \mathrm{s}^{-1}\right)$ was not too different from that observed in the 


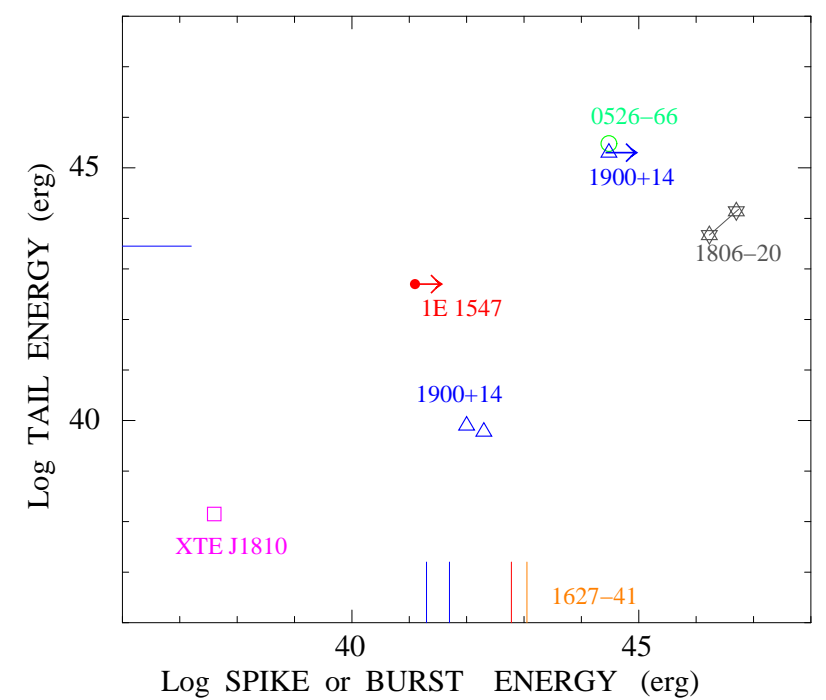

Figure 3: Energetics of flares and peculiar bursts from SGRs and AXPs. The ordinate gives the energy in the pulsating tails that often follow the brightest bursts, while the abscissa reports the energy in the initial spikes (data from [6]). The vertical/horizontal lines refer to events in which only one of these components has been observed. The three giant flares from SGRs are in the upper right corner. The two points for SGR 1806-20 are for the generally assumed distance of $15 \mathrm{kpc}$ and for the more recent estimate $\mathrm{d}=8.7 \mathrm{kpc}$ [14]. The energetics of the burst from 1E 1547.0-5408, for an assumed distance of $5 \mathrm{kpc}$, is in the range of the so called "intermediate flares".

pulsating tail of 1E $1547.0-5408\left(\sim 8 \times 10^{41}(\mathrm{~d} / 5 \mathrm{kpc})^{2} \mathrm{erg} \mathrm{s}^{-1}\right)$.

Comparison of the energy in the initial spikes is more difficult, since the effects of detector saturation and spectral uncertainties could be larger for these bright events. However, also in this case, it is clear that the initial spike of the 1E 1547.0-5408 burst involved a significantly smaller energy than those of the giant flares. From the point of view of the total energy, this event was more similar to the intermediate flares or to some of the brightest bursts observed in other SGRs, such as the 1998 October 28 [15] and the 2001 July 2 [16] events from SGR 1900+14, but with a shorter duration and higher peak luminosity. Another difference with respect to the three giant flares is that the latter had very short rise times [17, 18], while the initial spike of the 1E 1547.0-5408 burst showed a slow rise to the peak, that could be resolved in 4 ACS time bins (50 ms each).

The properties of the bursts from 1E 1547.0-5408 are typical of sources classified as SGRs. Indeed, if this AXP had not been previously known from X-ray and radio observations, it would have been named as a new SGR following the January 22 bursts. This underlines once more that the distinction between these two classes of neutrons stars is not based on physical properties of the sources, which are most likely explained by the same model.

\section{The dust scattering $X$-ray rings}

Soon after the discovery of the strong bursting activity of January 22, several follow-up pointings of 1E 1547.0-5408 were carried out with the Swift satellite. During the first Swift/XRT observations, the imaging mode could not be used because the source was too bright. The first data providing full imaging were obtained on January 23 at $\sim 15: 30$ UT and showed the presence of 

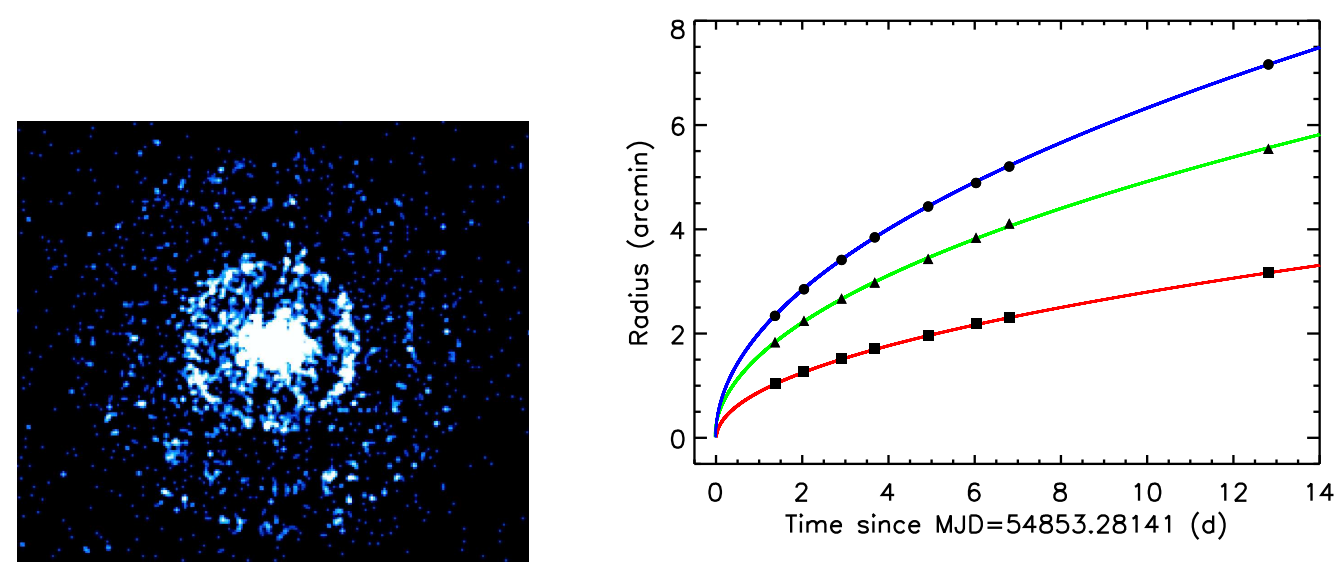

Figure 4: Left: X-ray rings produced by dust scattering around 1E 1547.0-5408 as observed with the Swift/XRT instrument on January 23. Right: Expansion of the three dust scattering rings (from [7]) fitted with a function $\theta(t)=K\left(t-t_{0}\right)^{1 / 2}$. The error bars are much smaller than the symbols.

remarkable dust scattering rings around the source position [19]. Further observations carried out with Swift, XMM-Newton and Chandra clearly showed that the angular size of the three rings increased with time (Fig. 4). The study of dust scattering X-ray halos around bright galactic sources can constrain the properties and spatial distribution of the interstellar dust. When the scattered radiation is a short burst/flare and the dust is concentrated in a relatively narrow cloud, an expanding ring (instead of a steady diffuse halo) appears, due to the difference in path-lengths at different scattering angles. X-ray expanding rings due to dust scattering have been observed in a few gamma-ray bursts, and through their study accurate distances of the scattering dust clouds in our galaxy could be determined [20, 21, 22].

The dust scattering rings around 1E 1547.0-5408 are the brightest ever observed and the first ones for an AXP/SGR. By fitting their expansion law we could determine the burst emission time, which is found to coincide with the interval of highest activity observed around 6:45 UT of January 22 (see Fig. 1). We have carried out a detailed analysis of the rings finding that a distance of 1E 1547.0-5408 in the range $\sim 4-5 \mathrm{kpc}$ is favored if the dust models that best fit several other observations are adopted [7].

\section{References}

[1] Thompson, C. \& Duncan, R. C., MNRAS, 275, 255, 1995

[2] Mereghetti, S., A\&AR, 15, 225, 2008

[3] Lamb, R. C. \& Markert, T. H., ApJ, 244, 94, 1981

[4] Gelfand, J. D. \& Gaensler, B. M., ApJ, 667, 1111, 2007

[5] Camilo, F., Ransom, S. M., Halpern, J. P., \& Reynolds, J., ApJ, 666, L93, 2007

[6] Mereghetti, S. et al., ApJ, 696, L74, 2009

[7] Tiengo, A., et al., ApJ, 710, 227, 2010 
[8] von Kienlin, A., Beckmann, V., Rau, A., et al., A\&A, 411, L299, 2003

[9] Kaneko, Y. and Göğüş, E. and Kouveliotou, C., et al., ApJ, 710, 1335, 2010

[10] Savchenko, V., Neronov, A., Beckmann, V., Produit, N., Walter, R. , A\&A, 510, 77, 2010

[11] Kaspi, V. M., Gavriil, F. P., Woods, P. M., et al., ApJ, 588, L93, 2003

[12] Israel, G. L., Romano, P., Mangano, V., et al., ApJ, 685, 1114, 2008

[13] Götz, D., Mereghetti, S., Molkov, S., et al., A\&A, 445, 313, 2006

[14] Bibby, J.L., et al., MNRAS, 386, L23, 2008

[15] Aptekar, R. L., Frederiks, D. D., Golenetskii, S. V., et al., ApJS, 137, 227, 2001

[16] Olive, J.-F., Hurley, K., Sakamoto, T., et al., ApJ, 616, 1148, 2004

[17] Mazets, E. P., Cline, T. L., Aptekar, R. L., et al., Astronomy Letters, 25, 635, 1999

[18] Schwartz, S. J., Zane, S., Wilson, R. J., et al., ApJ, 627, L129, 2005

[19] Tiengo, A., Mereghetti, S., Vianello, G., et al., GCN circ. 8848, 2009

[20] Vaughan, S., Willingale, R., O’Brien, P.T., et al., ApJ, 603, L5, 2004

[21] Tiengo, A. \& Mereghetti, S., A\&A, 449, 203, 2006

[22] Vianello, G., Tiengo, A., \& Mereghetti, S., A\&A, 473, 423, 2007 